\title{
Scaling of thermoelectric voltage induced by microwave radiation at the boundary between two-dimensional electron systems
}

\author{
N. Romero Kalmanovitz, I. Hoxha, Y. Jin, S. A. Vitkalov, and M. P. Sarachik \\ Physics Department, City College of the City University of New York, New York, New York 10031, USA \\ Ivan A. Larkin \\ International Center of Condensed Matter Physics, Brasília, Distrito Federal 70904-970, Brazil \\ T. M. Klapwijk \\ Department of Applied Physics, Delft University of Technology, 2628 CJ Delft, The Netherlands
}

(Received 24 October 2007; published 15 January 2008)

\begin{abstract}
We report measurements of the rectification of microwave radiation $(0.7-20 \mathrm{GHz})$ at the boundary between two-dimensional electron systems created by a narrow gap split gate on a silicon surface for different temperatures, electron densities, and microwave power. For frequencies above $4 \mathrm{GHz}$ and different temperatures, the rectified voltage $V_{d c}$ as a function of microwave power $P$ can be collapsed onto a single universal curve $V_{d c}^{*}=f^{*}\left(P^{*}\right)$ using two scaling parameters. The scaled voltage $V_{d c}^{*}$ is a linear function of power $P^{*}$ for small power and proportional to $\left(P^{*}\right)^{1 / 2}$ at higher power. A theory is developed which attributes the observed voltage to the thermoelectric response associated with local heating by the microwave radiation of adjacent twodimensional electron systems with different densities $n_{1}$ and $n_{2}$. Excellent quantitative agreement is obtained between theory and experiment.
\end{abstract}

DOI: 10.1103/PhysRevB.77.035415

PACS number(s): 73.40.Ei, 73.40.Jn, 73.50.Lw, 73.50.Pz

\section{INTRODUCTION}

The nonlinear behavior of low-dimensional electron systems has attracted a great deal of attention for its fundamental interest as well as for potentially important applications in nanoelectronics. In response to microwave radiation and dc bias, strongly nonlinear transport ${ }^{1-18}$ that gives rise to unusual states ${ }^{19-24}$ has been reported in two-dimensional systems of electrons in high magnetic field. There has also been great interest in the nonlinear response of quantum ballistic constrictions, where the effects of quantum interference, spatial dispersion, and electron-electron interactions play crucial roles. ${ }^{25-37}$

In this paper, we report a different type of nonlinearity of thermoelectric origin in a two-dimensional system of electrons. We have investigated the rectified $(\mathrm{dc})$ voltage induced by microwave radiation applied locally to the boundary between two-dimensional electron systems with different electron densities $n_{1}$ and $n_{2}$. A simple experimental geometry is used, in which closely spaced, electrically isolated gates give rise to a very large microwave field localized at the narrow slit between the gates, thereby enhancing the nonlinear response in the immediate vicinity of the gap. The gates are used to vary the electron densities separately and independently, providing a convenient and effective tool to control the strength of the nonlinearity. The rectified voltage $V_{d c}$ is found to be an odd function of the difference $\Delta n=n_{2}-n_{1}$ between the electron densities $n_{1}$ and $n_{2}$ of the two systems. Using two scaling parameters, all the data above $4 \mathrm{GHz}$ taken at different temperatures can be collapsed onto a single universal curve.

Excellent quantitative agreement is obtained with a theory that considers the local overheating of the electrons by microwaves near the narrow boundary between the two- dimensional (2D) systems, which gives rise to a voltage through the thermoelectric effect between two dissimilar two-dimensional metals. A fit of the experimental data to this theory yields an electron-phonon relaxation rate that is in good agreement with the relaxation rate obtained by a recent theory ${ }^{39}$ as well as other experiments. ${ }^{38}$

This experimental protocol provides an effective method for studying the thermoelectric properties of low-dimensional systems. An important advantage relative to other approaches ${ }^{38}$ is that the electron system is heated directly by the microwaves with negligible heating of the phonon system, thereby reducing the contribution of phonon drag to the thermoelectricity.

This paper is a continuation and expansion of research reported earlier. ${ }^{41,42}$ Although we attribute our observations to a thermoelectric effect, we use the term "microwave rectification," since we generate a dc voltage in response to (ac) microwave radiation. The paper is organized as follows: the experimental setup and measurements are described in the next section. The following section summarizes the experimental results. We then present a theory based on the thermoelectric effect. This is followed by a discussion of other possible sources for the observed rectification and a detailed comparison of data with the proposed theory. The paper ends with an overall summary of our findings.

\section{EXPERIMENTAL PROCEDURE}

The high-mobility $\mathrm{Si}$ metal-oxide-semiconductor fieldeffect transistors (Si-MOSFETs) $\left(\mu=2 \mathrm{~m}^{2} / \mathrm{V} \mathrm{s}\right.$ at $T=4 \mathrm{~K}$ and $n=5 \times 10^{15} \mathrm{~m}^{-2}$ ) used in these studies are equipped with several metallic gates that can be separately controlled. A narrow split obtained by reactive ion etching separates the different gates. Each 2D electron system is formed below a 


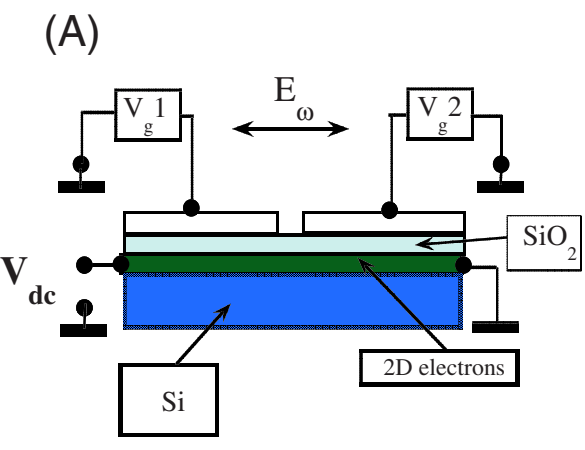

(B)

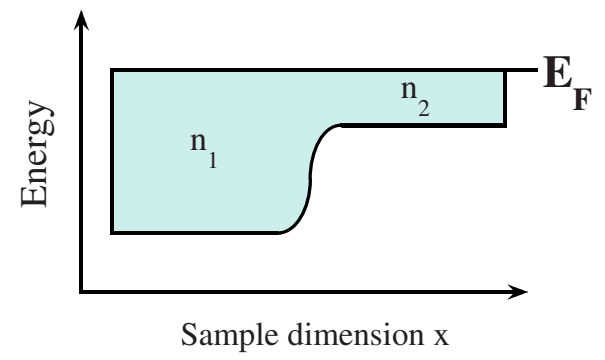

FIG. 1. (Color online) (A) Cross section of the sample. Two different 2D metals are formed under the two separate gates with voltages $V_{g} 1$ and $V_{g} 2$ applied as shown. The rectified dc voltage $V_{d c}$ is measured between the right and left ends of the structure (Ref. 44). The microwave voltage is applied directly to the gates and is localized near the slit between them (see Fig. 8). (B) The Fermi level and bottom of the conduction band are shown as a function of position $x$ along the sample. Energies below the Fermi level correspond to occupied electron states of the two 2D metals with different electron densities $n_{1}$ and $n_{2}$. The spatial variation of the electron density is described by Eq. (1).

rectangular $50 \times 240 \mu \mathrm{m}^{2}$ gate by the application of a positive voltage [see Fig. 1(A)]. The typical slit width of $50-70 \mathrm{~nm}$ is less than the thickness of the Si oxide insulating layer $(152 \mathrm{~nm})$, providing a smooth variation of the electron density between the two electron systems formed below the gates, as shown in Fig. 1(B). For a slit width $w$ that is much smaller than the distance $d$ between the gates and the two-dimensional electron gas (2DEG), the profile of electron density is given by ${ }^{43}$

$$
n(x)=\frac{n_{1}+n_{2}}{2}+\frac{n_{1}-n_{2}}{2} \tanh \left(\frac{\pi x}{d}\right),
$$

where $x$ is the distance from the center of the slit. For the actual parameters of our samples, the exact solution differs from Eq. (1) by less than 3\%. The six different pairs of 2D electron systems studied displayed similar behavior.

Measurements were taken at frequencies from 0.7 to $20 \mathrm{GHz}$ in a vacuum chamber of a He-3 cryostat. The microwave radiation was guided by a semirigid coaxial line terminated by a loop. Two wires, anchored to a temperature controlled cold finger, were inductively coupled to the loop without touching it. The wires were connected directly to the two adjacent gates. The sample, with a calibrated $\mathrm{RuO}_{2}$ ther- mometer attached, was thermally connected to the same cold finger. The temperature of the electrons was monitored using the amplitude of Shubnikov-de Haas oscillations. Without microwave power input, the electron temperature followed the temperature of the cold finger down to the lowest temperature $T=0.27 \mathrm{~K}$. The sample was, thus, well isolated from heat input deriving from the coaxial line and the rf filtered dc electrical leads.

Rather than the resistance, the dominant contribution to the output impedance $Z_{\text {out }}$ of the circuit at the end of the microwave line was provided by the (substantial) capacitive coupling between the two closely placed wires. Estimates indicated that variations of the resistivity of the $2 \mathrm{D}$ electrons with temperature and gate voltage have a negligibly small effect on $Z_{\text {out }}$. We, therefore, neglected the consequent small changes of output voltage $V_{a c}$. In particular, we neglected the effect of overheating the $2 \mathrm{D}$ electrons by the microwaves on the output impedance and consider the amplitude of the microwave voltage $V_{a c}$ applied to the gates to be proportional to the square root of the calibrated microwave power $P$ applied at the input of the coaxial line:

$$
V_{a c}=G P^{1 / 2},
$$

where $G$ is a temperature-independent coefficient.

The microwave-induced dc voltage was measured between two electrical contacts placed on opposite sides of the sample at a distance $L=240 \mu \mathrm{m}$ from the gap between the two electron systems [see Fig. 1(A)]. To avoid thermoelectric effects related to the electrical contacts, the distance $L$ must be considerably longer than the thermal relaxation length $L_{T}$, which is estimated to be $L_{T} \sim 100 \mu \mathrm{m}$ in Si-MOSFETs at temperatures of $\sim 1 \mathrm{~K}$ (Refs. 38, 39, and 45) (see Fig. 8). In this paper, we present data obtained at temperatures above $2 \mathrm{~K}$, where the contact thermoelectricity is negligibly small.

The same results for the rectification were obtained using continuous microwave radiation and by modulating the microwave amplitude at a frequency of typically $10 \mathrm{~Hz}$, and using standard phase sensitive techniques. All the data reported in this paper were obtained by the second method, as it provided better detection of signals below $1 \mu \mathrm{V}$.

\section{EXPERIMENTAL RESULTS}

\section{A. Dependence of the rectification on electron density}

Figure 2 shows the dependence of the rectified signal on the electron densities of the two adjacent 2D metals. The axes denote the electron densities $n_{1}$ and $n_{2}$, and the shading reflects the amplitude of the rectified signal, with dark (light) shading denoting negative (positive) values. Each horizontal scan was obtained for a fixed density $n_{1}$ [the electrons in the left-hand region of Fig. 1(A)], while the electron density $n_{2}$ in the adjacent (right hand) region is varied. Shown by the black dots in Fig. 2, the dc voltage changes sign when the electron densities of the two 2D metals are nearly the same.

We note that similar results were obtained in our previous experiments, ${ }^{41}$ where microwave radiation was applied by a very different method using two parallel wires placed far from the samples. This indicates that the results are robust 


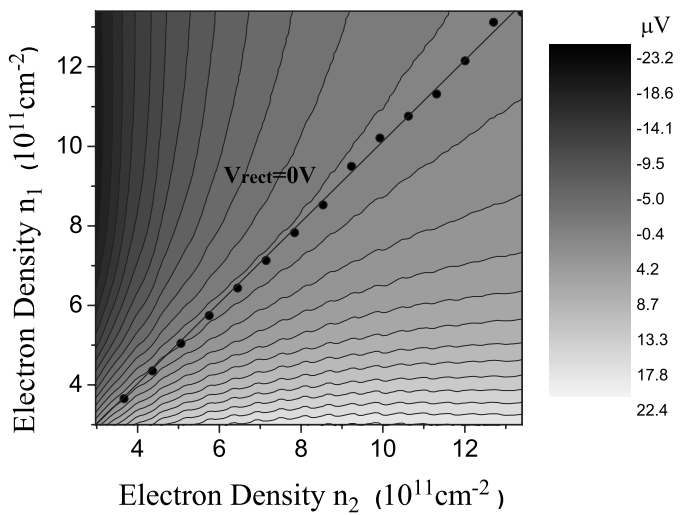

FIG. 2. "Topological map" of the rectified voltage as a function of the electron densities $n_{1}$ and $n_{2}$. The rectified voltage is indicated by the shading, ranging from dark to light as the voltage varies from negative to positive. Measurements were taken at a temperature of $4.2 \mathrm{~K}$, frequency of $20.2 \mathrm{GHz}$, and microwave power input of $26 \mathrm{~dB}$ (specified relative to $1 \mathrm{~mW}$ for $0 \mathrm{~dB}$ ). The rectification is an odd function of the difference between the two densities $n_{1}$ and $n_{2}$ [see Eq. (3)]. The black dots denote the values of $n_{1}$ and $n_{2}$ for which the rectified signal changes polarity (goes through zero).

and do not depend on the details of the distribution of electromagnetic fields in the vicinity of the sample, and that the dissimilarity between the two metals rather than the microwave field distribution is responsible for the effects observed. Moreover, the absence of rectification when $n_{1}=n_{2}$ is a strong experimental indication that bulk rectification inside the 2D metals, associated with microwave modulation of the electron density and/or mobility, is a minor contribution to the observed signal. This is discussed further in subsequent sections of the paper.

Figure 3 provides a clear demonstration that the rectified signal is an odd function of the difference $\Delta n=\left(n_{2}-n_{1}\right)$ between the electron densities of the two systems. Here, curve (a) shows the rectification when the electron density $n_{1}$ is fixed at $5.9 \times 10^{11} \mathrm{~cm}^{-2}$ while the electron density $n_{2}$ is varied; curve (b) is for fixed density $n_{2}=5.9 \times 10^{11} \mathrm{~cm}^{-2}$ and variable density $n_{1}$. Almost perfect antisymmetry is found with respect to the horizontal axis, showing that the rectification is an odd function of the difference between the two electron densities:

$$
V_{d c}\left(n_{1}-n_{2}\right)=-V_{d c}\left(n_{2}-n_{1}\right) .
$$

\section{B. Dependence of the rectification on microwave power at different temperatures}

Figure 4 shows the rectified voltage as a function of microwave power on a log-log scale for seven different temperatures ranging from 2.2 to $12 \mathrm{~K}$ at microwave frequency of $20 \mathrm{GHz} .{ }^{47}$ At all temperatures, the rectified signal for low power input is proportional to the microwave power (the square of the microwave electric field), $V_{d c} \propto P \propto E_{\omega}^{2}$. We will refer to this as the weak, or perturbative, nonlinear regime. Strongly nonlinear behavior is observed at higher levels of microwave excitation; here, the rectified signal is propor-

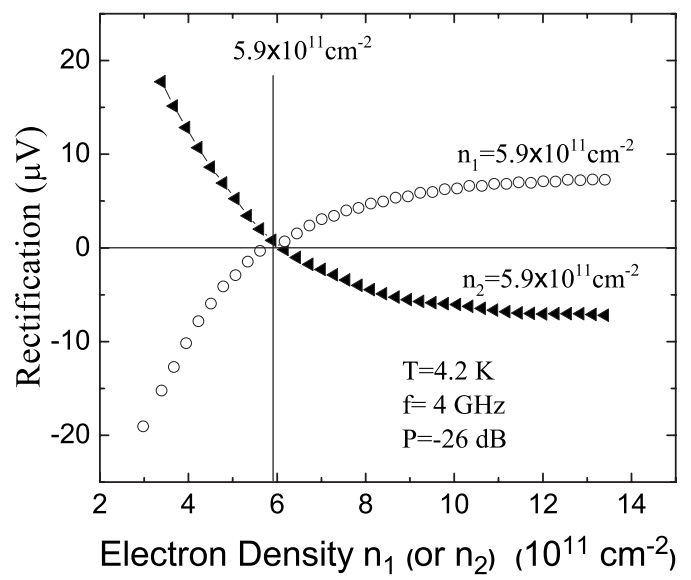

FIG. 3. The open circles denote the rectified signal as a function of the electron density $n_{2}$ with electron density $n_{1}$ kept constant at $5.9 \times 10^{11} \mathrm{~cm}^{-2}$. The triangles show the rectified signal versus the electron density $n_{1}$, with the density $n_{2}$ fixed at the same value of $5.9 \times 10^{11} \mathrm{~cm}^{-2}$. Measurements were taken at a temperature of $4.2 \mathrm{~K}$, frequency of $4 \mathrm{GHz}$, and microwave power of $26 \mathrm{~dB}$ (specified relative to $1 \mathrm{~mW}$ for $0 \mathrm{~dB}$ ). Note that if $n_{1}$ and $n_{2}$ are interchanged, the rectification changes sign and satisfies relation (3).

tional to the square root of the power, $V_{d c} \propto P^{1 / 2} \propto E_{\omega}$. The two straight lines drawn in Fig. 4 represent the two limits, namely, the perturbative $\left(V_{d c} \propto P\right)$ and the strongly nonlinear $\left(V_{d c} \propto P^{1 / 2}\right)$ regimes. The crossover between the two regimes depends on temperature, with the crossover occurring at higher microwave power as the temperature is increased.

By applying appropriate multiplicative scale factors (corresponding to translations on a log-log plot) to each of the curves of Fig. 4, one can collapse all the data for rectified voltage versus power at the frequency of $20 \mathrm{GHz}$ onto a single universal curve:

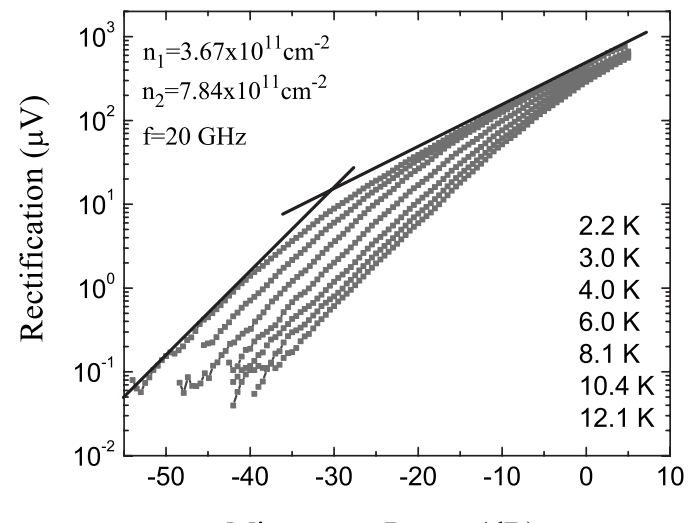

Microwave Power $(\mathrm{dB})$

FIG. 4. Rectified voltage as a function of microwave power on a $\log -\log$ scale. The units of power are specified relative to $0 \mathrm{~dB}$ for $1 \mathrm{~mW}$. Curves are shown for seven different temperatures ranging from $2.2 \mathrm{~K}$ (top curve) to $12.1 \mathrm{~K}$ (bottom curve). The straight lines represent linear $P$ and square root $P^{1 / 2}$ dependences of the rectification on microwave power $P$. The electron densities are $n_{1}=3.67$ $\times 10^{11} \mathrm{~cm}^{-2}$ and $n_{2}=7.84 \times 10^{11} \mathrm{~cm}^{-2}$. The microwave frequency is $20 \mathrm{GHz}$. 


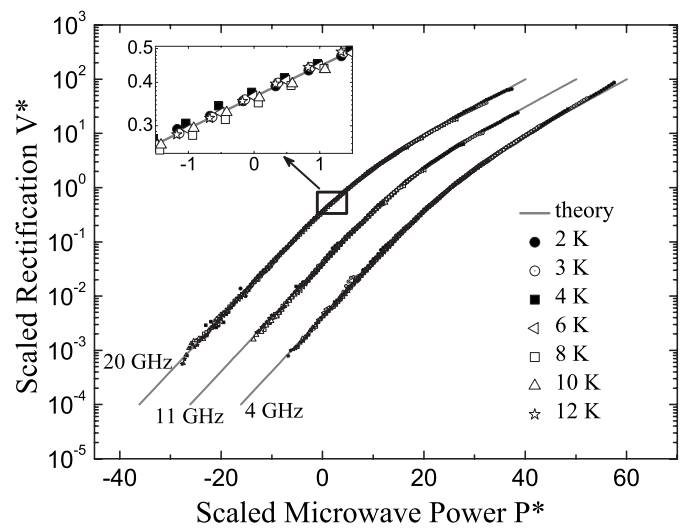

FIG. 5. Normalized rectified voltage $V_{d c}^{*}$ versus normalized microwave power $P^{*}$ for frequencies of $20 \mathrm{GHz}$ (the data of Fig. 4) $11 \mathrm{GHz}$, and $4 \mathrm{GHz}$ at different temperatures. The data for frequencies of 11 and $4 \mathrm{GHz}$ are shifted horizontally by 10 and $20 \mathrm{~dB}$, respectively, with respect to the curve at $20 \mathrm{GHz}$ for clarity. The solid lines are theoretical curves given by Eqs. (23) and (24) (also shifted by 10 and $20 \mathrm{~dB}$, respectively). The inset is a magnification of a portion of the top curve to indicate the quality of the scaling and/or typical deviations from the theory. A scaled curve is obtained at each frequency for all temperatures, and an appropriate horizontal shift brings the curves into alignment onto a single universal curve for all frequencies shown.

$$
V_{d c}^{*}=f^{*}\left(P^{*}\right)
$$

as shown in Fig. 5. Also shown in Fig. 5 are similarly scaled curves for frequencies of 11 and $4 \mathrm{GHz}$. Departures from scaling that occur below $4 \mathrm{GHz}$ will be discussed later in this paper.

The scaled values of the rectified voltage $V_{d c}^{*}=V / A(T)$ and power $P^{*}=P / B(T)$ require the two scaling parameters $A(T)$ and $B(T)$ shown in Fig. 6 as a function of temperature for different frequencies. For temperatures below $6 \mathrm{~K}$, the parameters $A(T) \propto T^{2}$ and $B(T) \propto T^{4}$, with deviations toward a weaker dependence at higher temperatures. There is no clear dependence on frequency. At a lower frequency $(0.7 \mathrm{GHz})$, the scaling breaks down for high power input, with substantial deviations from $V_{d c} \propto P^{1 / 2}$ behavior (not shown).

In the low power regime, the rectification is found to be proportional to microwave power at all frequencies. In this weakly nonlinear regime, the rectification can be written as

$$
V_{d c}=\alpha(T) \times P,
$$

where $\alpha(T)$ depends on the temperature. Figure 7 shows the constant of proportionality $\alpha$ plotted as a function of temperature for different frequencies.

The scaling behavior indicates (see Fig. 5) that $V_{d c}^{*}=\gamma P^{*}$ in the weak nonlinear regime with a constant, temperatureindependent $\gamma$. Since the scaling parameters $A$ and $B$ are given by $V_{d c}^{*}=V / A(T)$ and $P^{*}=P / B(T)$, it follows that the coefficient $\alpha=\gamma \frac{A(T)}{B(T)}$. The solid line shows the behavior expected from the theory in the scaling regime.

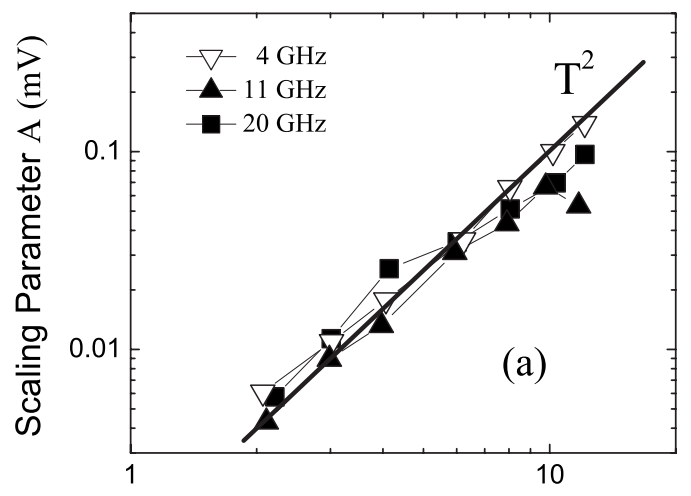

Temperature (K)

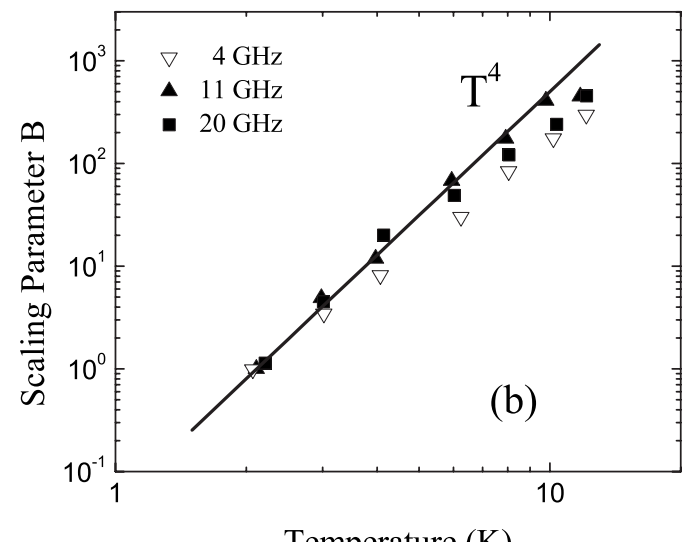

FIG. 6. (a) Dependence of scaling parameter $A$ on temperature at different frequencies, as labeled. The units of $A$ are chosen to conform to the theoretical prediction of Eq. (12). The solid straight line is the theoretical dependence corresponding to Eq. (13). (b) Dependence of scaling parameter $B$ on temperature at different frequencies, as labeled. The solid straight line is the theoretical dependence corresponding to Eq. (20) with the parameter $R \propto T_{L}^{6}$.

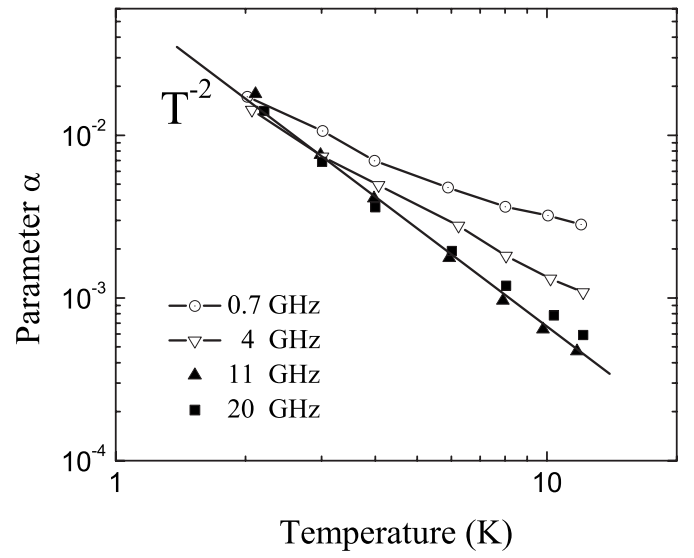

FIG. 7. Temperature dependence of the proportionality constant $\alpha$ in the low microwave power regime, where $V=\alpha \times P$. For 11 and $20 \mathrm{GHz}, \alpha \propto T^{-2}$; the straight line corresponds to the theoretical prediction [Eqs. (12) and (19)] at high frequencies. The electron densities are $n_{1}=3.67 \times 10^{11} \mathrm{~cm}^{-2}$ and $n_{2}=7.84 \times 10^{11} \mathrm{~cm}^{-2}$. 


\section{THEORY}

In this section, we present a quantitative theory for the microwave rectification in the two $2 \mathrm{D}$ electron systems studied in the previous section. In this theory, the dc voltage results from the thermoelectric effect induced by local microwave overheating of the area near the boundary between two dissimilar 2D electron metals. First, we present a system of electrodynamic equations which allows us to find the distribution of microwave electric potential across the sample. We will show that at high frequency, the microwave field is localized near the narrow gap between the metals. Then we solve the thermoconductivity equation assuming fast thermalization of hot 2D electrons and find the temperature profile inside the electron system. Deviations from the isotropic Fermi distribution should not significantly affect the spatial relaxation and, therefore, will not be discussed in this paper. The thermoelectric voltage $V_{d c}$ is found by direct integration of the thermoelectric field across the sample.

In the derivation of the microwave (ac) current distribution, we take into account (i) that the microwave wavelength $(0.7-30 \mathrm{~cm})$ is much larger than the device size $(\sim 0.05 \mathrm{~cm})$ and, therefore, we can omit the term $\partial B / \partial t$ in Maxwell's equations; and (ii) that the scale at which the electric potential varies $l_{a c}$ is much larger than the effective distance of the 2D conducting layer from the gates $d$. Together with the law of electric charge conservation, the full set of equations for the time-dependent current distribution $j(x, t)$, density $\delta n(x, t)$, and the electric potential at the 2D conducting plane reads:

$$
\begin{gathered}
j(x, t)=\sigma(x) \nabla \phi(x, t), \\
\partial_{t}[e \delta n(x, t)]=-\nabla j(x), \\
\phi(x, t)=\phi_{0}(x, t)+\frac{e d}{\epsilon \epsilon_{0}} \delta n(x, t),
\end{gathered}
$$

where $\sigma(x)$ is the local conductivity, $\epsilon$ is the dielectric constant of $\mathrm{SiO}_{2}$, and $\phi_{0}(x, t)=(1 / \pi) V_{a c} \arctan (x / d) \cos (\omega t){ }^{48}$

At high frequencies, $\omega / 2 \pi>10 \mathrm{GHz}$, the major part of the microwave power is absorbed by the 2 DEG in the narrow strip under the slit between the two gates. The size of the narrow region (hot strip) is

$$
l_{a c}=\left(\frac{2 \sigma d}{\omega \epsilon \epsilon_{0}}\right)^{1 / 2} .
$$

Figure 8 shows the distribution of microwave power (solid line) obtained by a numerical solution of Eqs. (6)-(8). The microwave power is found to be localized near the slit.

The electron temperature distribution obeys the thermoconductivity equation:

$$
\nabla(\kappa \nabla T(x))=F(T(x))-W_{a c}(x),
$$

where $\kappa$ is thermoconductivity coefficient, $W_{a c}(x)$ is Joule heat, and $F(T)$ stands for the power losses. The result of numerical integration of Eq. (10) together with Eqs. (6)-(8) gives a temperature distribution shown schematically in Fig. 8 by the dashed line. We use $F(T)=R(T)-R\left(T_{L}\right)$ for the

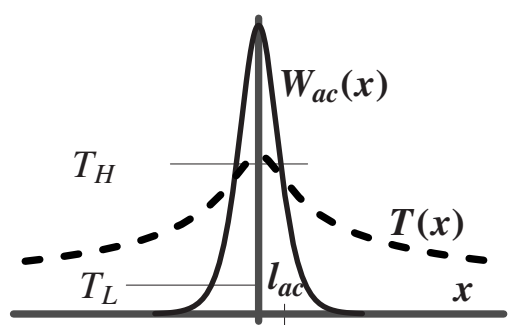

FIG. 8. Joule input (solid line) and electron temperature (dashed line) vs distance $x$ from the center of the hot strip.

power losses due to phonons, ${ }^{38}$ where $R(T)$ is the electronphonon relaxation rate and $T_{L}$ is the lattice temperature, which we assume to be unaffected by the microwaves. Figure 8 shows that the Joule heat decreases exponentially from the slit as $\exp \left[-2 x / l_{a c}\right]$, while the electron temperature relaxes much more slowly as $\left(x / L_{T}\right)^{-1 / 2}$.

At zero dc current, the temperature gradient and electric field are related by ${ }^{49}$

$$
E(x)=Q(T) \nabla T(x),
$$

where the thermopower coefficient $Q=\frac{1}{3} \pi^{2} \beta T / e E_{F}$ with numerical coefficient $\beta \sim 1{ }^{50}$ The electric potential difference between the two contacts is found by integrating the left and right sides of Eq. (11) over the distance $x$ :

$$
\begin{gathered}
V_{d c}=A\left(T_{L}\right) \cdot(y(0)-1), \\
A\left(T_{L}\right)=\frac{\pi^{2} T_{L}^{2}}{6 e}\left(\frac{\beta_{L}}{E_{F L}}-\frac{\beta_{R}}{E_{F R}}\right),
\end{gathered}
$$

where we have introduced a dimensionless parameter $y(x)$ $=\left[T(x) / T_{L}\right]^{2}$. The indexes $L$ and $R$ correspond to the left and right sides of the $2 \mathrm{DEG}$, and $T(x)$ is the temperature of the hot electrons along the sample. The coefficient $A$ is antisymmetric with respect to the difference between Fermi energies in the left and right regions and is, thus, antisymmetric with respect to the difference of electron densities $\Delta n=n_{R}-n_{L}$. This behavior is consistent with the experimental observations (see Fig. 3).

At low ac power, a small electron overheating $\Delta T=T(0)$ $-T_{L}$ is proportional to the microwave power $P$ and the linear dependence of $V_{d c}$ on the microwave power follows from Eq. (12). In the high power regime, the voltage $V_{d c}$ is determined by the electron temperature below the slit $T(0)$, which, in turn, depends nonlinearly on the microwave power. The crossover from the linear regime to the nonlinear regime occurs at $\left[T(0)-T_{L}\right] / T_{L} \sim 1$. Note that if $l_{a c}$ is much less than the sample size, then Eqs. (12) and (13) hold independently of the model for the thermoconductivity and the power loss function $F(T)$. Therefore, the only parameter that determines the voltage $V_{d c}$ is the temperature $T(0)$ of the $2 \mathrm{D}$ electrons in the hot strip under the slit.

To find the temperature profile, we solve the thermoconductivity equation (10). Since in our setup the phonon system is not directly heated by the microwave radiation, the phonon temperature is weakly affected by the microwaves. 
To compare theory with the experimental findings, both the phonon heat transport and the phonon drag contribution to the thermopower are neglected. To proceed further, we use the Wiedemann-Franz law for the electron thermoconductivity $\kappa(T)=\pi^{2} \sigma T / 3 e^{2}$. When $x \gg l_{a c}$, we can reduce the order of the differential equation (10) by neglecting the second term on the right-hand side (Joule heat), yielding

$$
\left(\frac{\pi^{2} \sigma T_{L}^{2}}{12 e^{2}}\right)\left(\frac{d y}{d x}\right)^{2}=R\left(T_{L}\right)\left(\frac{1}{4} y^{4}-y+\frac{3}{4}\right) .
$$

Experiments ${ }^{38}$ have shown that in the temperature range from 1 to $5 \mathrm{~K}$,

$$
R(T)=R_{1} T^{6},
$$

where $R_{1}$ is a constant. For the density $8.5 \times 10^{11} \mathrm{~cm}^{-2}, R_{1}$ $\approx 1.3 \mathrm{~mW} \mathrm{~m}^{-2} \mathrm{~K}^{-6}$. According to the theory, ${ }^{39} R(T)$ deviates weakly from the $T^{6}$ law and depends on electron concentration as $n^{-1 / 2}$. The temperature relaxation length can be expressed in terms of $\kappa(T)$ and $R(T)$ as

$$
L_{T}=\sqrt{\frac{T \kappa(T)}{R(T)}} .
$$

The total Joule heat of the 2DEG $W_{a c}^{T}$ is given by

$$
W_{a c}^{T}=\int \overline{j(x, t) \nabla \phi(x, t) d x}=\eta V_{a c}^{2}\left(\frac{\sigma_{L}}{l_{a c}^{L}}+\frac{\sigma_{R}}{l_{a c}^{R}}\right),
$$

where the upper bar denotes average over time and $\eta \sim 1$ is a numerical factor.

At a stationary state, the input heat is partially absorbed by the phonon system and partially drained via the electron thermal flow. When $l_{a c} \ll L_{T}$, most of the input power generated in the hot strip, $-l_{a c}<x<l_{a c}$, must be drained away via the thermal electron flow, because the hot strip is much smaller than the total overheated area $\left(\sim L_{T}\right)$ absorbing the total heat input. In this case, neglecting the phonon absorption in the hot strip area, a simple integration of Eq. (10) over the region $-l_{a c}<x<l_{a c}$ leads to

$$
W_{a c}^{T}=\frac{\pi^{2} T_{L}^{2}}{6 e^{2}}\left[\sigma_{L}\left(\frac{d y}{d x}\right)_{L}-\sigma_{R}\left(\frac{d y}{d x}\right)_{R}\right] .
$$

Combining Eqs. (14), (17), (18), (9), and (2), we obtain a relation between the microwave input power $P$ and the temperature of the 2D electrons under the slit as $y(0)$ $=\left[T(0) / T_{L}\right]^{2}$ :

$$
\begin{gathered}
P=B(T) \cdot\left[y(0)^{4}-4 y(0)+3\right]^{1 / 2}, \\
B(T)=\frac{\sqrt{3} \pi}{6 \eta G^{2}}\left(\frac{T_{L}^{2} R\left(T_{L}\right) d}{e^{2} \omega \epsilon \epsilon_{0}}\right)^{1 / 2} .
\end{gathered}
$$

These equations, together with Eq. (12), determine the dependence of the rectified voltage $V_{d c}$ on the lattice temperature $T_{L}$ and microwave power $P$. Expanding Eq. (19) at small $P$, we get

$$
y(0)=1+\frac{\eta \sqrt{2} P}{\pi G^{2}}\left(\frac{T_{L}^{2} R\left(T_{L}\right) d}{e^{2} \omega \epsilon \epsilon_{0}}\right)^{-1 / 2}
$$

and for the weakly nonlinear regime $[y(0)-1 \ll 1]$

$$
V_{d c}=\frac{A\left(T_{L}\right) P}{\sqrt{6} B\left(T_{L}\right)} \sim \frac{L_{T}}{l_{a c}} \frac{e V_{a c}^{2}}{E_{F}} .
$$

One can see that, expressed in normalized values of the $\mathrm{dc}$ voltage $V_{d c}^{*}=V_{d c} / A(T)$ and the input microwave power $P^{*}$ $=P / B(T)$, the system of Eqs. (12) and (19) exhibits a universal form in the whole range of microwave power:

$$
\begin{gathered}
V_{d c}^{*}=y(0)-1, \\
P^{*}=\left[y(0)^{4}-4 y(0)+3\right]^{1 / 2} .
\end{gathered}
$$

The universal dependence $V_{d c}^{*}$ vs $P^{*}$ is plotted in Fig. 5, together with the scaled data points. Excellent agreement is found between the experiment and the theory in a broad range of temperature and microwave power. Random deviations between the experiment and the theory observed at low power are mostly due to low signal/noise ratio for signals below $1 \mu \mathrm{V}$.

To conclude this section, we consider other mechanisms that may also lead to rectification of an ac voltage. We first consider the bulk rectification associated with the fact that $\sigma$ in Eq. (6) may vary due to microwave modulation of the electron density and/or mobility inside the $2 \mathrm{DEG} .{ }^{30,46}$ To estimate this effect, we assume that the bulk rectification is due to a periodic variation of the electron density induced by the microwave modulation of the gate voltage $V_{g}: \delta n$ $=n V_{a c} / V_{g} \cdot{ }^{30}$ The induced dc current $I_{d c}^{b u l k}=\sigma V_{d c}^{b u l k}=\delta \sigma V_{a c}$. Therefore, $V_{d c}^{b u l k}=\frac{\delta \sigma}{\sigma} V_{a c}=\frac{\delta n}{n} V_{a c}=V_{a c}^{2} / V_{g}$. This is considerably smaller than the rectification due to the thermoelectric effect: From Eq. (22), $V_{d c} \sim V_{a c}^{2} / E_{F}$, as $E_{F} \ll V_{g}$. Comparison of $V_{d c}^{b u l k}$ with result (22) shows that $V_{d c}^{\text {bulk }} \sim 2 \times 10^{-4} V_{d c}$.

Another possible mechanism of rectification is related to the spatial variation of the electron density across the boundary. ${ }^{51}$ Due to this variation, there is a diffusive electron flow through the boundary. The net flow of electrons must be zero at thermodynamic equilibrium. An internal electric field $E_{b}$ is established to compensate the diffusive flow across the boundary, creating a so-called contact potential difference. Microwave radiation moves the electron system away from thermodynamic equilibrium. The nonequilibrium (symmetric) part of the distribution function is driven by the internal electric field $E_{b}$, creating a rectified current and rectified voltage $V_{d c}^{b}$. This voltage is estimated to be $V_{d c}^{b}$ $\sim\left(\tau^{2} e / m\right) \ln \left(n_{1} / n_{2}\right) E_{\omega}^{2}$, where $\tau$ is the relaxation time, assumed in Ref. 51 to be on the same order of magnitude as the transport relaxation time, $m$ is the band mass of the $2 \mathrm{D}$ electrons, and $E_{\omega} \sim V_{a c} / l_{a c}$ is the ac electric field near the boundary. Using our result for $l_{a c}$, we get 


$$
V_{d c}^{b} \sim \frac{1}{e n_{1,(2)} d} \tau \omega V_{a c}^{2} \ln \left(\frac{n_{1}}{n_{2}}\right) .
$$

Comparison of $V_{d c}^{b}$ with Eq. (22) shows that $V_{d c}^{b} \sim 5$ $\times 10^{-4} V_{d c}$, and is inconsistent with the significant temperature dependence observed in the experiment.

\section{DISCUSSION}

In the preceding sections, we reported measurements of the rectification of microwave radiation at the boundary between two-dimensional electron systems separated by a narrow $\sim 110 \mathrm{~nm}$ transition region created by independently controlled gates on a silicon surface. The rectified signal is large, it is odd with respect to the interchange of the gates, and, within a broad range of temperatures and frequencies, all data for the rectified voltage versus microwave input power collapse onto a single universal curve using two scaling parameters.

The fact that interchanging the gates gives rise to a signal that is essentially the same in magnitude and of opposite sign indicates that bulk rectification of the incoming microwave signal is a small contribution. Contrary to expectations for bulk rectification, the observed signal changes sign, does not depend on the detailed geometry of the sample and gates, and does not depend on the size of the sample. Moreover, as shown in Sec. IV, we estimate that bulk rectification would contribute a signal that is considerably smaller than the mechanism we propose. It should be noted further that our observations cannot be attributed to rectification by nonOhmic contacts, as this would yield a signal that depends on the microwave field distribution along the sample and, therefore, should not be simply antisymmetric when the gates are interchanged.

The near-perfect antisymmetry of the rectification on interchanging gates, the fact that the microwave power is strongly localized near the boundary between the 2D metals, and the excellent agreement with theory, all provide strong evidence that the observed rectification is an inherent property of the two adjacent 2D electron systems of different densities. In particular, we attribute the observed rectification to a thermoelectric response due to strong local overheating of the electron gas that produces a large thermal gradient at the gap between the gates, where the electron density changes abruptly. We now proceed to present a detailed comparison between this theory and the experimental results.

As shown in Fig. 5, data for the rectified voltage versus input power can be collapsed over a broad range of temperature and microwave power onto a single universal curve using two scaling parameters. Plots of Eqs. (23) and (24) are shown by the solid line. Excellent agreement is obtained between theory and experiment in a broad range of temperatures and microwave power.

The scaling coefficients $A(T)$ and $B(T)$ used to obtain the data collapse vary with temperature, as shown in Fig. 6. The temperature dependence of parameter $A(T)$ denoted by the solid line in Fig. 6(a) is proportional to $T^{2}$, in agreement with Eq. (13). At $T=3 \mathrm{~K}$, the scaling parameter $A(3 \mathrm{~K})=10 \mu \mathrm{V}$, in good agreement with the theoretical estimate obtained us- ing the Fermi energy $E_{F}$ and the parameter $\beta=0.2$ for $n_{1}$ $=3.67 \times 10^{11} \mathrm{~cm}^{-2}$ for Si-MOSFETs. ${ }^{40}$ At higher temperatures $(\sim 10 \mathrm{~K})$, the experimental values of $A$ tend to fall below that of the theory because the relaxation length $L_{T}$ is short, so that the approximation $L_{T} \gg l_{a c}$ made in the theory is no longer valid. We also note that for electron density $n_{1}$ $=3.67 \times 10^{11} \mathrm{~cm}^{-2}$, the Fermi energy in Si-MOSFETS is about $20 \mathrm{~K}$, so that at $T \sim 10 \mathrm{~K}$, the electron system is not strongly degenerate and finite temperature corrections to the thermoelectric coefficient $Q$ [see Eq. (11)] and the Wiedemann-Franz ratio have to be taken into account.

Surprisingly, the theory gives much better scaling than one would expect from the accuracy of both the thermopower coefficient $Q$ and the Wiedemann-Franz relation. This may reflect the fact that acoustic phonon scattering is quasielastic and hot electrons remain in the "energy quasiballistic" regime. ${ }^{52}$ A careful analysis of the electron kinetics is required to verify this.

The scaling parameter $B(T)$ is shown in Fig. 6(b). The parameter $B$ displays similar behavior as a function of temperature for all measured frequencies above $4 \mathrm{GHz}$. The temperature dependence is due to the strong dependence of the power losses $F(T)=R_{1}\left(T^{6}-T_{L}^{6}\right)$ on the lattice temperature. The solid line shows the theoretical expectation using an approximation of the power losses by Eq. (15) derived from recent theory ${ }^{39}$ and experiment. ${ }^{38}$ At higher temperatures, discrepancies between theory and experiment are seen, which are most likely associated with deviations from scaling at low frequencies, for reasons discussed below.

Figure 7 shows the temperature dependence of the parameter $\alpha$, the constant of proportionality that relates the rectified voltage $V_{d c}$ to the microwave power $P$ in the weakly nonlinear regime [see Eq. (5)]. According to the theory [see Eq. (22)], the coefficient $\alpha$ is proportional to the ratio of the scaling parameter $A$ to the parameter $B: \alpha(T) \propto A(T) / B(T)$ $\propto 1 / T^{2}$; the theoretically expected behavior is shown in the figure by the solid straight line. Good agreement with theory is obtained at high microwave frequencies $(>10 \mathrm{GHz})$, where the microwave radiation is well localized near the boundary between the two 2D metals. However, progressively stronger deviations from the theory develop as the frequency is decreased. These deviations correlate with deviations from scaling observed at frequencies below $4 \mathrm{GHz}$.

In particular, at a frequency of $0.7 \mathrm{GHz}$, the rectification does not depend on power as $P^{1 / 2}$ at high power, and the universality expressed in Eqs. (23) and (24) is not observed. We suggest that these deviations from theoretical expectations are due to the fact that the experimental results are outside the range of validity of the theory in its present form. Analytical and numerical estimates indicate that at low frequency ( $1 \mathrm{GHz}$ and below), the microwave field is barely localized near the boundary between the two 2D metals. The corresponding size of the hot strip at the frequency of $1 \mathrm{GHz}$ is $l_{a c} \sim 80 \mu$, which is considerably broader than the temperature relaxation length $L_{T}$, especially in the high temperature domain (several microns at $T>6 \mathrm{~K}$ ). For these conditions, one of the central approximations of the theory $\left(l_{a c}\right.$ $\left.\ll L_{T}\right)$ is no longer valid. 


\section{SUMMARY}

We report systematic investigations of the dc voltage generated at the boundary between adjacent two-dimensional electron systems subjected to microwave radiation. Measurements were taken over a broad range of temperature, electron density, microwave power, and microwave frequency. For microwave frequencies between 4 and $20 \mathrm{GHz}$, the highest frequency measured in these experiments, the induced dc voltage versus microwave power can be collapsed onto a single universal curve, $V_{d c}^{*}=f^{*}\left(P^{*}\right)$, using two parameters. Over the range investigated in these experiments, the scaling exhibits two different power regimes. For small power, the voltage is a linear function of power, $V_{d c}^{*} \propto P^{*}$, while at higher power, the rectification is proportional to $\left(P^{*}\right)^{1 / 2}$. Quantitative agreement is found with a theory that attributes the observed voltage to the thermoelectric response associated with local heating by the microwave radiation of two adjacent two-dimensional electron systems with different densities $n_{1}$ and $n_{2}$. A fit of the experimental data to the theory yields a temperature dependence for the electronphonon relaxation rate that is in good agreement with the temperature dependence obtained by a recent theory ${ }^{39}$ as well as other experiments. ${ }^{38}$ In addition, the fit gives the dimensionless constant in the expression for thermopower for a degenerate $2 \mathrm{D}$ electron gas that is in reasonable agreement with theory ${ }^{50}$ and experiment. ${ }^{53}$

\section{ACKNOWLEDGMENTS}

The work at the City College of New York was supported by DOE Grant No. DOE-FG02-84-ER45153. The work at the International Center of Condensed Matter Physics, Brasília, was supported by the IBEM fund from the Brazilian Ministry of Science and Technology.
${ }^{1}$ M. A. Zudov, R. R. Du, J. A. Simmons, and J. R. Reno, Phys. Rev. B 64, 201311(R) (2001).

${ }^{2}$ P. D. Ye, L. W. Engel, D. C. Tsui, J. A. Simmons, J. R. Wendt, G. A. Vawter, and J. L. Reno, Appl. Phys. Lett. 79, 2193 (2001).

${ }^{3}$ S. I. Dorozhkin, JETP Lett. 77, 577 (2003).

${ }^{4}$ R. L. Willett, L. N. Pfeiffer, and K. W. West, Phys. Rev. Lett. 93, 026804 (2004).

${ }^{5}$ S. A. Studenikin, M. Potemski, A. Sachrajda, M. Hilke, L. N. Pfeiffer, and K. W. West, Phys. Rev. B 71, 245313 (2005).

${ }^{6}$ A. A. Bykov, A. K. Bakarov, D. R. Islamov, and A. I. Toropov, JETP Lett. 84, 391 (2006).

${ }^{7}$ C. L. Yang, J. Zhang, R. R. Du, J. A. Simmons, and J. L. Reno, Phys. Rev. Lett. 89, 076801 (2002).

${ }^{8}$ A. A. Bykov, Jing-qiao Zhang, Sergey Vitkalov, A. K. Kalagin, and A. K. Bakarov, Phys. Rev. B 72, 245307 (2005).

${ }^{9}$ Jing-qiao Zhang, Sergey Vitkalov, A. A. Bykov, A. K. Kalagin, and A. K. Bakarov, Phys. Rev. B 75, 081305(R) (2007).

${ }^{10}$ W. Zhang, H.-S. Chiang, M. A. Zudov, L. N. Pfeiffer, and K. W. West, Phys. Rev. B 75, 041304(R) (2007).

${ }^{11}$ A. C. Durst, S. Sachdev, N. Read, and S. M. Girvin, Phys. Rev. Lett. 91, 086803 (2003).

${ }^{12} \mathrm{P}$. W. Anderson and W. F. Brinkman, arXiv:cond-mat/0302129 (unpublished).

${ }^{13}$ J. Shi and X. C. Xie, Phys. Rev. Lett. 91, 086801 (2003).

${ }^{14}$ M. G. Vavilov and I. L. Aleiner, Phys. Rev. B 69, 035303 (2004).

${ }^{15}$ I. A. Dmitriev, M. G. Vavilov, I. L. Aleiner, A. D. Mirlin, and D. G. Polyakov, Phys. Rev. B 71, 115316 (2005).

${ }^{16}$ J. Alicea, L. Balents, M. P. A. Fisher, A. Paramekanti, and L. Radzihovsky, Phys. Rev. B 71, 235322 (2005).

${ }^{17}$ M. G. Vavilov, I. L. Aleiner, and L. I. Glazman, Phys. Rev. B 76, 115331 (2007).

${ }^{18}$ I. A. Dmitriev, A. D. Mirlin, and D. G. Polyakov, Phys. Rev. B 75, 245320 (2007).

${ }^{19}$ W. Zhang, M. A. Zudov, L. N. Pfeiffer, and K. W. West, Phys. Rev. Lett. 98, 106804 (2007).

${ }^{20}$ A. V. Andreev, I. L. Aleiner, and A. J. Millis, Phys. Rev. Lett. 91, 056803 (2003).

${ }^{21}$ A. Auerbach, I. Finkler, B. I. Halperin, and A. Yacoby, Phys. Rev.
Lett. 94, 196801 (2005).

${ }^{22}$ R. G. Mani, V. Narayanamurti, K. von Klitzing, J. H. Smet, W. B. Jonson, and V. Umansky, Nature (London) 420, 646 (2002).

${ }^{23}$ M. A. Zudov, R. R. Du, L. N. Pfeiffer, and K. W. West, Phys. Rev. Lett. 90, 046807 (2003).

${ }^{24}$ A. A. Bykov, Jing-qiao Zhang, Sergey Vitkalov, A. K. Kalagin, and A. K. Bakarov, Phys. Rev. Lett. 99, 116801 (2007).

${ }^{25}$ L. DiCarlo, C. M. Marcus, and J. S. Harris, Jr., Phys. Rev. Lett. 91, 246804 (2003).

${ }^{26}$ Jiang Wei, Michael Shimogawa, Zenghui Wang, Iuliana Radu, Robert Dormaier, and David Henry Cobden, Phys. Rev. Lett. 95, 256601 (2005).

${ }^{27}$ R. Leturcq, D. Sanchez, G. Gotz, T. Ihn, K. Ensslin, D. C. Driscoll, and A. C. Gossard, Phys. Rev. Lett. 96, 126801 (2006).

${ }^{28}$ D. M. Zumbuhl, C. M. Marcus, M. P. Hanson, and A. C. Gossard, Phys. Rev. Lett. 96, 206802 (2006).

${ }^{29}$ A. Lofgren, C. A. Marlow, I. Shorubalko, R. P. Taylor, P. Omling, L. Samuelson, and H. Linke, Phys. Rev. Lett. 92, 046803 (2004).

${ }^{30}$ J. Q. Zhang, S. Vitkalov, Z. D. Kvon, J. C. Portal, and A. Wieck, Phys. Rev. Lett. 97, 226807 (2006).

${ }^{31}$ L. Angers, E. Zakka-Bajjani, R. Deblock, S. Gueron, A. Cavanna, U. Gennser, M. Polianski, and H. Bouchiat, Phys. Rev. B 75, 115309 (2007).

${ }^{32}$ P. W. Brouwer, Phys. Rev. B 63, 121303(R) (2001).

${ }^{33}$ M. G. Vavilov, V. Ambegaokar, and I. L. Aleiner, Phys. Rev. B 63, 195313 (2001).

${ }^{34}$ D. Sanchez and M. Buttiker, Phys. Rev. Lett. 93, 106802 (2004).

${ }^{35}$ B. Spivak and A. Zyuzin, Phys. Rev. Lett. 93, 226801 (2004).

${ }^{36}$ M. L. Polianski and M. Buttiker, Phys. Rev. Lett. 96, 156804 (2006).

${ }^{37}$ A. V. Andreev and L. I. Glazman, Phys. Rev. Lett. 97, 266806 (2006).

${ }^{38}$ R. Fletcher, V. M. Pudalov, Y. Feng, M. Tsaousidou, and P. N. Butcher, Phys. Rev. B 56, 12422 (1997).

${ }^{39}$ A. Sergeev, M. Yu. Reizer, and V. Mitin, Phys. Rev. Lett. 94, 136602 (2005). 
${ }^{40}$ V. K. Karavolas, M. J. Smith, T. M. Fromhold, P. N. Butcher, B. G. Mulimani, B. L. Gallagher, and J. P. Oxley, J. Phys.: Condens. Matter 2, 10401 (1990).

${ }^{41}$ I. Hoxha, S. A. Vitkalov, N. A. Zimbovskaya, M. P. Sarachik, and T. M. Klapwijk, arXiv:cond-mat/0110331 (unpublished).

${ }^{42}$ I. A. Larkin, S. A. Vitkalov, and M. P. Sarachik, Braz. J. Phys. (to be published).

${ }^{43}$ J. H. Davies and I. A. Larkin, Phys. Rev. B 49, 4800 (1994).

${ }^{44}$ The data reported in subsequent figures of this paper were obtained for a sample in which the gates, electrical contacts, and microwave electric field are aligned as shown in Fig. 1, so that the dc and microwave electric fields are parallel. Similar results were obtained for $\lambda$-shaped gate configurations, where the dc electric field changes direction between the contacts.

${ }^{45}$ O. Prus, M. Reznikov, U. Sivan, and V. Pudalov, Phys. Rev. Lett. 88, 016801 (2001).

${ }^{46}$ V. I. Falko, Sov. Phys. Solid State 31, 561 (1989).
${ }^{47}$ For temperatures $T<4 \mathrm{~K}$, the data were obtained in a small magnetic field of $0.4 \mathrm{~T}$ to suppress superconductivity of the aluminum gates and avoid the change (about 20-30\%) of the microwave field $E_{\omega}$ near the sample due to a superconducting transition (Ref. 41).

${ }^{48}$ J. H. Davies, I. A. Larkin, and E. V. Sukhorukov, J. Appl. Phys. 77, 4504 (1995).

${ }^{49}$ A. A. Abrikosov, Fundamentals of the Theory of Metals (Elsevier Science, New York, 1988).

${ }^{50}$ V. C. Karavolas and P. N. Butcher, J. Phys.: Condens. Matter 2, 3947 (1990).

${ }^{51}$ N. A. Zimbovskaya, Phys. Rev. B 68, 113404 (2003).

${ }^{52}$ Yu. Dubrovskii, I. A. Larkin, and S. Morosov, Superlattices Microstruct. 8, 233 (1991).

${ }^{53}$ R. Fletcher, V. M. Pudalov, and S. Cao, Phys. Rev. B 57, 7174 (1998). 